\title{
La fracción libre del antigeno prostático especifico en la detección de cáncer de prostata
}

Catalona W, Partin A, Slawin K et al. Use of percentage of free prostate-specific antigen to enhance differentiation of prostate cancer from benign prostatic disease. JAMA 1998; 279:1542-1547

\author{
Objetivo \\ Determinar un punto de corte clínicamente útil para el dosaje de anti- \\ geno prostático específico libre (APE-L). Evaluar si el APE-L tiene me- \\ jor especificidad que el APE total en la detección de cáncer de prósta- \\ ta $(C P)$, en pacientes con aumentos moderados del APE total. \\ Diseño \\ Ensayo clínico multicéntrico, aleatorizado, ciego y prospectivo (en \\ $9 \%$ de las muestras de sangre retrospectivo). \\ Lugar \\ Siete centros universitarios de EEUU.

\section{Pacientes} \\ Un total 773 pacientes de 50 a 75 años (mediana 64) con tacto recta \\ normal, con moderada elevación del APE total $(4-10 \mathrm{ng} / \mathrm{ml}$, mediana \\ $5,8)$, sin tratamiento, y con biopsia confirmatoria del diagnóstico his- \\ tológico. Seleccionaron 379 con CP y 394 con enfermedad prostática \\ benigna, para obtener el número de pacientes necesarios para reali- \\ zar el estudio.
}

\section{Descripción del test en estudio}

Dosaje en suero del APE-L por técnica de anticuerpos monoclonales

Descripción de test de referencia

Biopsia prostática en 6 tomas guiada por ecografía, previa a la determinación en suero del APE-L. Los patólogos que interpretaban la biopsia estaban ciegos al APE del paciente.

\section{Medición de reultados principales}

Curvas ROC* de APE-L comparado con el APE total en pacientes con APE total entre 4 y $10 \mathrm{ng} / \mathrm{ml}$. A partir de ella se seleccionaron puntos de corte para obtener una sensibilidad del $95 \%$. Se hizo una correción estadística para que la prevalencia de CP no sea $50 \%$ sino $25 \%$, que es la frecuencia previamente reportada de CP en el grupo de pacientes con esos valores de APE total. De esta manera se pudo calcular el valor predictivo clínicamente aplicable del test.

\section{Resultados Principales}

El área bajo la curva* para el APE-L fue de 0,72 vs. 0,52 para el APE total (en el rango mencionado). Un punto de corte de $25 \%$ de APE-L produjo una sensibilidad de $95 \%$ y una especificidad de $20 \%$ para el diagnóstico de CP. El APE-L se correlacionó en forma directa con la edad $(r=0,34)$, con el tamaño prostático ecográfico $(r=0,55)$; e inver-samente con el APE total $(r=-0,14)$.

\section{Conclusiones}

En pacientes con valores moderadamente aumentados de APE total y tacto rectal normal; el APE- $\mathrm{L} \leq 25 \%$ tiene una sensibilidad del $95 \%$ y una especificidad del $20 \%$ para CP (este $20 \%$ sería el porcentaje de biopsias que se evitarían de usarse el test en la población mencionada).

\section{COMENTARIO}

El objetivo de este estudio es determinar el valor de corte del APE-L con una mínima pérdida de la sensibilidad, evitando asi biopsias innecesarias en los pacientes con APE-L normal. La población en estudio incluye 773 hombres entre 50 y 75 años en quienes el tacto rectal fue normal y los valores de PSA total oscilaron entre 4 y $10 \mathrm{ng} / \mathrm{ml}$. Todos los pacientes fueron sometidos a una biopsia y a la determinación del porcentaje de APE-L.

Los autores cuidaron algunos factores que pueden influenciar los resultados obtenidos. El rango del APE total no fue amplio para evitar la distinta prevalencia de cáncer que se observa en estas circunstancias. El tacto rectal era normal en todos los pacientes. Esto tiene importancia pues el valor predictivo positivo* del APE total con tacto sospechoso de cáncer es el doble que el de aquellos con tacto normal. La muestra del estudio parece ser lo suficientemente amplia.

Con un valor de corte del APE-L del $25 \%$ o menos la sensibilidad es del $95 \%$, es decir que se "pierden" de diagnosticar el $5 \%$ de los cánceres. La especificidad con este valor de corte (porcentaje de biopsias que se podrían ahorrar) es del $20 \%$.

Relacionando los valores de corte con la edad observaron que la sensibilidad aumentaba en las personas más jóvenes. Así entre los 50 y 59 años la sensibilidad fue del $98 \%$ mientras que entre los 69 y 75 años fue del $90 \%$. Esto tiene importancia, por que en teoría se subdiagnosticarían más cánceres en personas mayores donde la expectativa de vida es menor y el comportamiento del tumor es menos agresivo. Asi mismo se diagnosticarían mejor a las personas jóvenes con mayor expectativa de vida y tumores más agresivos.

El objetivo del uso del APE-L es disminuir el número de biopsias innecesarias, sin disminuir la sensibilidad; y el valor de corte de hasta un $25 \%$ parece ser el adecuado.

Este estudio debe ser considerado en el contexto de la controversia actual acerca del rastreo de CP ${ }^{1}$. Mientras las guías preventivas en atención primaria (US Preventive Task Force, Canadian Task Force) concluyen que no hay suficiente evidencia a favor ni en contra para recomendar el rastreo con APE, tacto rectal o ecografía (recomendación tipo $C^{*}$ ), las recomandaciones de los especialistas (American Urologic Association, American Cancer Society) adhieren al rastreo con APE y tacto rectal anual en mayores de 50 años $^{2}$. Varios estudios randomizados de gran escala se estan llevando a cabo para intentar responder este interrogante ${ }^{3}$. Hoy en día los esfuerzos en CP deben estar orientados a demostrar la mejoría de la calidad de vida o sobrevida con un tratamiento temprano de la enfermedad.

Si uno toma la controvertida decisión del rastreo luego de hablar sobre riesgos, beneficios y preferencias de nuestros pacientes, el APE-L puede ser útil en aquellos pacientes con APE total moderadamente elevado para evitar biopsias innecesarias. También es importante tener en cuenta otros antecedentes del paciente, el tacto rectal, al valor de APE y su evolución en el tiempo. Solo así podrían disminuir los costos del tan controvertido rastreo de cáncer de próstata.

*Ver Glosario

\section{Dr. Carlos Giudice (h).}

Servicio de Urología. Hopital Italiano. 